and particles with matter, the luminescence process, photoelectricity and secondary emission. From there it proceeds to more technical items, such as the performance of commercial photomultipliers, the properties of scintillating materials and the preparation of crystals. The last chapters contain a survey of representative applications, including the direct counting of particles with open multipliers, and a brief discussion of various problems concerning the associated electronic circuits. Future developments and still unexplored possibilities are hinted at in many instances.

The author was certainly confronted with a thorny task in trying to bring order and proportion into this multitude of different topics. He has not shirked the countless obstacles on his way, but sometimes has not quite sueceeded in surmounting them; his choice of emphasis or perspective is often unconvincing, and the great compression of material has led to a high stylistic density which makes the book difficult to read. That the two chapters on luminescence should be the weakest part of the whole can scarcely invite criticism, for it seems well-nigh impossible to give an adequate survey of so intricate and controversial a subject in merely forty pages. But-to touch only a few points - there is no need to digress on the ray-tracing methods of electron optics if afterwards no example is discussed of actual electron trajectories in one of the more common multiplier types; it confuses a delicate issue if the statistical fluctuations in the height of scintillation pulses are treated in connexion with the signal-to-noise ratio pertinent to cinema and television work; and in the section on crystal-growing, the sulphides and tungstates, with their attractive properties, would have deserved more than a few scant remarks. The inquisitive reader could have been helped here or there by the inclusion of references to suitable review articles, but several of the most competent ones are not mentioned.

Despite these shortcomings, the book will be most welcome as an introductory guide to prospective users of the scintillation counter, and as a handy manual to many research workers, bringing together, as it does, for the first time all the multifarious aspects of an exceedingly complex device.

ERnst Breitenberger

\section{MODERN PHYSICS FOR DEGREE STUDENTS}

An Introduction to Thermodynamics, the Kinetic Theory of Gases, and Statistical Mechanics By Prof. Francis Weston Sears. Second edition. (Principles of Physics Series.) Pp. $\mathrm{x}+374$. (Cambridge, Mass.: Addison-Wesley Publishing Company, Inc., 1953.) 7.50 dollars.

7 HIS text-book, written by the professor of physics at the Massachusetts Institute of Technology, constitutes one of the volumes of the "Principles of Physics Series". It is so eminently readable, however, that it will appeal not only to the physics student but equally to the engineering student who wishes to extend his reading beyond the limits of most engineering texts on thermodynamics.

The book brings together, at degree level, the fundamental principles of thermodynamics, kinetic theory and statistical mechanics. The first half deals with classical thermodynamics. An introductory chapter is followed by a discussion of the equation of state and $p-v-T$ surfaces of ideal and real gases. The sections on the first and second laws of thermodynamics, and their consequences, are separated by a chapter on changes of phase, in which the forms of the $p-v-T$ surfaces for real substances are presenter in a number of excellent three-dimensional diagrams. These are a particular feature of the book. In a chapter on the combined first and second laws, the engineer will find a very lucid treatment of the difference between the ice point and the triple point of water, but will not agree with the statement that his steam tables are based on the former as the datum state point. A very brief chapter on some engineering applications of thermodynamics concludes this first section.

The second half of the book is devoted about equally to sections on kinetic theory and statistical mechanics. In the first of these, the derivation of the equation of state of an ideal gas, and a brief discussion of the Clausius and van der Waals equations of state, are followed by the determination of the Maxwell distribution function, and a chapter on the transport phenomena of viscosity, thermal conductivity and diffusion.

The concluding section follows the historical order in the presentation of statistical mechanics, dealing first with the application of the Maxwell-Boltzmann statistics to gases, with a paragraph on the specific heat capacity of solids, followed by a section on the elementary theory of a paramagnetic gas.

In the section on quantum statistics, the BoseEinstein distribution function is derived, but is applied only to the two special cases of an ordinary gas and a photon gas, to show that the former application leads again to the Maxwell--Boltzmann distribution function, and the latter gives the Planck distribution of energy density of radiation with frequency. The Fermi-Dirac statistics for an electron gas are then discussed, but are applied quantitatively only to the conditions at absolute zero of temperature, with a statement of the results obtained at temperatures other than this. A final chapter deals with the theory of fluctuations, and its applications to the scattering of light, to Brownian motion and to background noise in amplifiers.

The limitation of the application of statistical mechanics to the simpler cases results in an exceptionally readable introduction to a difficult subject, in which the physical implications of the mathematical manipulations are always clearly brought out. At the end of each chapter is a selection of problems with the answers provided. The MKS system of units is used throughout. The book is well produced, and suffers only from a number of minor misprints.

R. W. HAYWOOD

\section{AMERICAN SCIENCE WRITING}

Scientific American Reader

Pp. xiv +626 . (New York: Simon and Schuster, 1953.) 6 dollars.

JOW in its 108th year, the journal Scientific 1 American was taken over five years ago by a small group of science journalists who were committed to the belief that there is a growing interest in clear and exact reporting of contemporary scientific events. Their skill, energy and enthusiasm has led to success, and to-day the journal has a circulation in excess of 125,000 . 